\title{
Flip-Top Dispensing Plastic Container Closure
}

National Cancer Institute

\section{Source}

National Cancer Institute. Flip-Top Dispensing Plastic Container Closure. NCI Thesaurus.

Code C96128.

A hing ed single or dual flap closure for controlled product dispensing. 